\title{
Review Board
}

Avril Aitken, Bishop's University

Susann Allnutt, McGill University

Abigail Anderson, Ministère de I'Éducation, du Loisir et du Sport

Glenda L. Bissex, University of Vermont Jon Bradley, McGill University

Faith Butler, College of the Bahamas

David Dillon, McGill University

Jean Fillatre, Lester B. Pearson School

Board

Corrine Glesne, University of Vermont

Rita Irwin, University of British

Columbia

Joanne Kingsley, Bishop's University

Neomi Kronish, Education Consultant

Carl Leggo, University of British

Columbia

Mary Maguire, McGill University

Heather McBride, Education

Consultant

Matthew Meyer, St. Francis Xavier

University

Shaun Murphy, University of

Saskatchewan

Joe Norris, Brock University

Marni Pierce, Alberta Children and

Youth Initiative
Stefinee Pinnegar, Brigham Young

University

Tiiu Poldma, Université de Montréal

Debbie Pushor, University of

Saskatchewan

Lori Rabinovitch, Education Consultant

J. Kenneth Robertson, Champlain

Regional College

Alissa Sklar, Concordia University

Sylvia Sklar, McGill University

Lauren Small, New Frontiers School

Board

Carolyn Sturge Sparkes, Memorial

University

Teresa Strong-Wilson, McGill University

Teri Todd, California State University

Chico

Kathleen Vaughan, Concordia

University

Evelyn Vingilis, University of Western

Ontario

Sandra Weber, Concordia University

Boyd White, McGill University

Lee Williams, Slippery Rock University 